\title{
Bifurcation of a Prey-predator System with Holling Type-III Functional Response Function
}

\author{
Ji Zhang ${ }^{1}$, Xiaofei Zhang ${ }^{2}$ \\ ${ }^{1}$ Tianjin University of Technology and Education No.1310,Dagu South Road, Hexi District, Tianjin 300222, China \\ ${ }^{2}$ Vishay General Semiconductor Co., Ltd. NO.88 Sixth Street, Tianjin Economic and Technological Development Area, China
}

\begin{abstract}
We consider a predator-prey system with generalized Holling type-III functional response. The results developed in this article reveal far richer dynamics compared to the model without Holling type-III functional response. The model have two equilibrium in the interior for some values of parameters. We perform a qualitative analysis of model which includes positivity of solutions local asymptotic stability and bifurcation of equilibrium and analyze the local stability properties of the equilibrium of the model through the standard linearization technique. The main purpose of the article is to offer a relatively complete mathematical analysis for the model.
\end{abstract}

Keywords: prey-predator system, holling type-III functional response, bifurcation

\section{Introduction}

The purpose of this section is to propose a prey-predator mode:

$$
\left\{\begin{array}{l}
\frac{d x}{d t}=r x\left(1-\frac{x}{K}\right)-\frac{c_{1} x y}{m_{1}+x}, \\
\frac{d y}{d t}=s y\left(1-\frac{c_{2} y}{m_{2}+x}\right)
\end{array}\right.
$$

Let $x(t)$ and $y(t)$ denote densities of the prey and predators respectively at time $t$.The coefficients $r$ and $K$ represent intrinsic growth rate and environmental carrying capacity for the prey in the absence of predation. The growth rate of the predator under natural conditions by $s$, while $s c_{2}$ is the maximum value of the pre capita reduction rate of predators. $c_{1}$ is the maximum value of the per capita reduction rate of prey, while $m_{1}$ and $m_{2}$ represent the extent to which the environment provides protection to the prey and predator, respectively. All the parameters are assumed to be positive due to biological considerations.

System (1) has been studied by many scholars [1-8], A prey-predator model with (predator harvesting) Holling type-III is studied in this paper. Using the scaling we obtain the following system of differential equations [9];

$$
\left\{\begin{array}{l}
\frac{d x}{d t}=x\left(1-x-\frac{\alpha y}{m+x}\right) \equiv x f^{(1)}(x, y), \\
\frac{d y}{d t}=\rho y\left(1-\frac{\beta y}{m+x}-\delta y\right) \equiv y f^{(2)}(x, y),
\end{array}\right.
$$

with the initial conditions

$$
x(0)=x_{0}>0, y(0)=y_{0}>0
$$

Here

$$
f^{(1)}(x, y)=1-x-\frac{\alpha y}{m+x}, f^{(2)}(x, y)=\rho\left(1-\frac{\beta y}{m+x}-\delta y\right)
$$

We are only interested in the dynamics of system (2) in the first quadrant from the standpoint of biology.

$$
R_{0}^{+} \times R_{0}^{+}=\left\{(x, y) \in R^{2} \mid x \geq 0, y \geq 0\right\}
$$

\section{Positivity of solution}

Lemma 2.1 For all $t>0$, all solution $(x(t), y(t))$ of (2) with initial condition (3) are positive.

Proof. System (2) gives

$$
x(t)=x(0) \exp \left(\int_{0}^{t} f^{(1)}(x(s), y(s)) d s\right)>0,
$$

and

$$
y(t)=y(0) \exp \left(\int_{0}^{t} f^{(2)}(x(s), y(s)) d s\right)>0,
$$

With initial condition (3), hence all solution starting from an interior of first quadrant remain in it for all future time.

\section{Existence and stability of axial equilibrium}

In order to find $t$ growth isoc he equilibrium of system (2), we consider the zero lines of the system,

$$
x f^{(1)}(x, y)=0, y f^{(2)}(x, y)=0
$$

The equilibrium are now the point of intersection of these zero growth isoclines. The axial equilibrium of system (2) are

$s_{0}=(0,0), s_{1}=(1,0) s_{2}=(0, m /(\beta+\delta m))$.

Lemma 3.1 (i) The origin $s_{0}=(0,0)$ is always a unstable point.

(ii) The axial equilibrium $s_{1}=(1,0)$ is always a saddle point.

(iii) The axial equilibrium $s_{2}=(0, m /(\beta+\delta m))$ is a stable if $\alpha>\beta+\delta m$ and a saddle point if $\alpha<\beta+\delta m$.

Proof. The Jacobin matrix of system (2) is given by

$$
J=\left(\begin{array}{cc}
1-2 x-\frac{\alpha m y}{(m+x)^{2}} & \frac{-\alpha x}{m+x} \\
\frac{\rho \beta y^{2}}{(m+x)^{2}} & \rho-\frac{2 \rho \beta y}{m+x}-2 \rho \delta y
\end{array}\right)
$$

So

$$
J_{0}=\left(\begin{array}{ll}
1 & 0 \\
0 & \rho
\end{array}\right)
$$




\section{International Journal of Science and Research (IJSR) \\ ISSN (Online): 2319-7064}

Index Copernicus Value (2013): 6.14 | Impact Factor (2014): 5.611

The eigenvalues of $J_{0}$ are $\lambda_{1}=1$ and $\lambda_{2}=\rho$

$$
J_{1}=\left(\begin{array}{cc}
-1 & \frac{-\alpha}{m+1} \\
0 & \rho
\end{array}\right)
$$

The eigenvalues of $J_{1}$ are $\lambda_{1}=-1$ and $\lambda_{2}=\rho$

$$
J_{2}=\left(\begin{array}{cc}
1-\frac{\alpha}{\beta+\delta m} & 0 \\
\frac{\rho \beta}{(\beta+\delta m)^{2}} & -\rho
\end{array}\right),
$$

$\lambda_{1}=1-\alpha /(\beta+\delta m)$ and $\lambda_{2}=-\rho$ are the eigenvalues of $J_{2}$.Therefore the result follows.

\section{Bifurcation of interior equilibrium}

In this section, we study the bifurcation of co-existing equilibrium which are important form an ecological point view. $S_{1^{*}}=\left(x_{1^{*}}, y_{1^{*}}\right)$ and $S_{2^{*}}=\left(x_{2^{*}}, y_{2^{*}}\right)$ are the interior equilibrium points, where $x_{1^{*}}$ and $x_{2^{*}}$ are the positive root of the quadratic equation:

$$
\delta x^{2}+(\beta+m \delta-\delta) x+(\alpha-\beta-\delta m)=0,
$$

That is

$$
x_{1^{*}}=\frac{(\delta-\beta-m \delta)-\sqrt{\Delta}}{2 \delta}, x_{2^{*}}=\frac{(\delta-\beta-m \delta)+\sqrt{\Delta}}{2 \delta}
$$

and

$$
y_{1^{*}}=\frac{\left(1-x_{1^{*}}\right)\left(m+x_{1^{*}}\right)}{\alpha}, y_{2^{*}}=\frac{\left(1-x_{2^{*}}\right)\left(m+x_{2^{*}}\right)}{\alpha} .
$$

together with

$$
\Delta=(\beta+\delta m-\delta)^{2}-4 \delta(\alpha-\beta-\delta m)
$$

Case I: If $\alpha>\beta+\delta m,(\beta+\delta m-\delta)^{2}<4 \delta(\alpha-\beta-\delta m)$ then no interior equilibrium exists.

Case II: if $\alpha>\beta+\delta m$ and $(\beta+\delta m-\delta)^{2}=4 \delta(\alpha-\beta-\delta m)$ $\beta+\delta m<\delta$, then the equation (6) has a multiple root, say $\bar{x}=\frac{(\delta-\beta-m \delta)}{2 \delta}$ and $\bar{y}=\frac{(1-\bar{x})(m+\bar{x})}{\alpha}$. Hence system (2) has an instantaneous interior eauilibrium $\bar{S}=(\bar{x}, \bar{y})$.

Case III: if $(\beta+\delta m-\delta)^{2}>4 \delta(\alpha-\beta-\delta m)$ and $\beta+\delta m<\delta$ $S_{1^{*}}=\left(x_{1^{*}}, y_{1^{*}}\right)$ and $S_{2^{*}}=\left(x_{2^{*}}, y_{2^{*}}\right)$ as the two distinct interior equilibria.

Let

$$
G(x)=2 \delta x^{2}+(\beta+3 \delta m-\delta) x+\left(m^{2} \delta+m \beta-m \delta\right)
$$

We know

$$
G^{\prime}(x)=4 \delta x+\beta+3 \delta m-\delta
$$

That is

$$
M=\frac{\delta-\beta-3 \delta m}{4 \delta}
$$

Theorem 4.1 $0 \leq M<x_{1^{*}}<\bar{x}<x_{2^{*}}<1$ and $\beta+\delta m<\delta$

(i) The equilibriu $S_{1^{*}}=\left(x_{1^{*}}, y_{1^{*}}\right)$ is always a saddle point .

(ii) The equilibriu $S_{2^{*}}=\left(x_{2^{*}}, y_{2^{*}}\right)$ is always a stable point if

$$
\rho>\frac{\alpha\left(x_{2^{*}}-2 x^{2}{ }_{2^{*}}-m x_{2^{*}}\right)}{\left(m+x_{2^{*}}\right)\left(1-x_{2^{*}}\right)\left(\beta+\delta m+\delta x_{2^{*}}\right)} .
$$

(iii) System (2) undergoes a Hopf-bifurcation around the equilibriu $S_{2^{*}}=\left(x_{2^{*}}, y_{2^{*}}\right)$ if

$$
\rho=\frac{\alpha\left(x_{2^{*}}-2 x_{2^{*}}^{2}-m x_{2^{*}}\right)}{\left(m+x_{2^{*}}\right)\left(1-x_{2^{*}}\right)\left(\beta+\delta m+\delta x_{2^{*}}\right)} .
$$

Theorem $4.20<x_{1^{*}}<\bar{x}<x_{2^{*}}<M$ and $\beta+\delta m<\delta$

(i) The equilibriu $S_{2^{*}}=\left(x_{2^{*}}, y_{2^{*}}\right)$ is always a saddle point .

(ii) The equilibriu $S_{1^{*}}=\left(x_{1^{*}}, y_{1^{*}}\right)$ is always a stable point if

$$
\rho>\frac{\alpha\left(x_{2^{*}}-2 x_{2^{*}}^{2}-m x_{2^{*}}\right)}{\left(m+x_{2^{*}}\right)\left(1-x_{2^{*}}\right)\left(\beta+\delta m+\delta x_{2^{*}}\right)} .
$$

(iii) System (2) undergoes a Hopf-bifurcation around the equilibriu $S_{1^{*}}=\left(x_{1^{*}}, y_{1^{*}}\right)$ if

$$
\rho=\frac{\alpha\left(x_{2^{*}}-2 x_{2^{*}}^{2}-m x_{2^{*}}\right)}{\left(m+x_{2^{*}}\right)\left(1-x_{2^{*}}\right)\left(\beta+\delta m+\delta x_{2^{*}}\right)}
$$

Proof. According to the proof of the theorem 3.1, we have theorem 4.1 and 4.2.

Theorem 4.3 System(2) undergoes a saddle-node bifurcation around $\bar{S}=(\bar{x}, \bar{y})$ with respect to bifurcation parameter $\alpha$ with bifurcation threshold $\alpha^{[s n]}$, if : $\beta+\delta m<\delta$ and $\rho>\frac{\alpha\left(\bar{x}-2 \bar{x}^{2}-m \bar{x}\right)}{(m+\bar{x})(1-\bar{x})(\beta+\delta m+\delta \bar{x})},(\beta+\delta m-\delta)^{2}=4 \delta(\alpha-\beta-\delta m)$.

Proof. The Jacobian $\bar{J}$ at the equilibrium $\bar{S}=(\bar{x}, \bar{y})$ is given by

$$
\bar{J} \equiv D g(\bar{x}, \bar{y})=\left(\begin{array}{cc}
\frac{\bar{x}(1-\bar{x})}{m+\bar{x}}-\bar{x} & \frac{-\alpha \bar{x}}{m+\bar{x}} \\
\frac{\rho \beta(1-\bar{x})^{2}}{\alpha^{2}} & -\rho \bar{y}\left(\frac{\beta}{m+\bar{x}}+\delta\right)
\end{array}\right)
$$

We know

$$
\bar{x}=\frac{(\delta-\beta-m \delta)}{2 \delta}, \bar{y}=\frac{(1-\bar{x})(m+\bar{x})}{\alpha}
$$

We also see that

if

$\alpha=\alpha^{[s n]}=\frac{(\beta+\delta+\delta m)^{2}}{4 \delta} \rho>\frac{\alpha\left(\bar{x}-2 \bar{x}^{2}-m \bar{x}\right)}{(m+\bar{x})(1-\bar{x})(\beta+\delta m+\delta \bar{x})}$

We have

$$
\bar{J}=\left(\begin{array}{cc}
\frac{\beta \bar{x}}{\delta(m+\bar{x})} & \frac{-\alpha \bar{x}}{m+\bar{x}} \\
\frac{\rho \beta}{\alpha \delta} & -\rho
\end{array}\right)
$$

so

$$
\begin{gathered}
\operatorname{det} \bar{J}=\frac{-\rho \beta \bar{x}}{\delta(m+\bar{x})}-\frac{(-\alpha \bar{x}) \rho \beta}{\alpha(\delta m+\bar{x})}=0 \\
\operatorname{tr} \bar{J}=\frac{\beta \bar{x}}{\delta(m+\bar{x})}-\rho<\frac{\beta \bar{x}}{\delta(m+\bar{x})}-\frac{\alpha(\bar{x}-2 \bar{x}-m \bar{x})}{(m+\bar{x})(1-\bar{x})(\beta+\delta m+\delta \bar{x})}=0
\end{gathered}
$$




\section{International Journal of Science and Research (IJSR) \\ ISSN (Online): 2319-7064}

Index Copernicus Value (2013): 6.14 | Impact Factor (2014): 5.611

The eigenvalue of the Jacobin at the saddle-node equilibrium Must be zero and the other eigenvalue must have negative real part. Therefore we can use Sotomayor's theorem. Let

$$
g=\left(g^{(1)}, g^{(2)}\right)
$$

There

$$
g^{(1)} \equiv x f^{(1)}, g^{(2)} \equiv x f^{(2)}
$$

Let $V=\left(v_{1}, v_{2}\right)^{T}$ and $W=\left(w_{1}, w_{2}\right)^{T}$ be the eigenvectors of $\bar{J}$ and $\bar{J}^{T}$ corresponding to the zero eigenvalue, A simple calculation yields

$$
\begin{gathered}
V=\left(\frac{\alpha \delta}{\beta}, 1\right)^{T} \\
W=\left(\frac{\rho \delta(1-x)^{2}(x+m)}{\alpha^{2} x},-1\right)^{T}=\left(\frac{\rho(x+m)}{\alpha x},-1\right)^{T}
\end{gathered}
$$

Therefore,

Since

$$
\Omega_{1}=W^{T} g_{\alpha}\left(\bar{S}, \alpha^{[s n]}\right)=\frac{\rho \delta \bar{y}^{2}}{(\delta+\beta+\delta m)(m+x)}<0
$$

$$
\begin{gathered}
g_{\alpha}\left(\bar{S}, \alpha^{[s n]}\right) \equiv \frac{\partial g}{\partial \alpha}\left(\bar{S}, \alpha^{[s n]}\right)=\left(\begin{array}{c}
-\frac{\bar{x} \bar{y}}{m+\bar{x}} \\
0
\end{array}\right) \\
\Omega_{2}=\mathrm{W}^{\mathrm{T}}\left[D^{2} g\left(\bar{S}, \alpha^{[s n]}\right)(V, V)\right]=-\frac{2 \rho}{x y}-\frac{\rho}{\beta x y}[(3 m-1)(\delta+\beta+\delta m-1)]<0
\end{gathered}
$$

We also see that

$$
D^{2} g\left(\bar{S}, \alpha^{[s n]}\right)(V, V)=\left(\begin{array}{c}
V^{T}\left(\begin{array}{cc}
f_{x x}^{(1)} & f_{x y}^{(1)} \\
f_{y x}^{(1)} & f_{y y}^{(1)}
\end{array}\right) V \\
V^{T}\left(\begin{array}{ll}
f^{(2)} x x & f^{(2)} x y \\
f^{(2)} & f^{(2)} y y
\end{array}\right) V
\end{array}\right)
$$

Thus from Sotomayor's theorem, system(2) undergoes a saddle-node bifurcation around $\bar{S}=(\bar{x}, \bar{y})$.

\section{Conclusion}

In this paper, we considered a biological prey -predator model with Holling type-III functional response. It is observed that the model have two equilibrium in the interior of the positive prey-predator plane. The stability of the equilibrium has been discussed. There are two kinds of interior equilibrium which is locally stable for certain parametric restrictions. Saddle node and Hopf-bifurcation are also exhibited in this paper. There results indicate that prey-predator with nonlinear functional response not only depend on bifurcation parameters but also are so sensitive to parameter perturbations that it is important for the control of infections diseases or biological species. Finally the analysis provides a certain method for understanding biological information and carrying on the related intelligent computation.

\section{References}

[1] B. Leard, C. Lewis and J. Rebaza, Dynamics of ratio-dependent predator-prey models with nonconstant harvesting, Discrete Contin.Dyn.Syst.Ser.S,1(2008), 303-315 Liu Liqun. Reverse engineering and finite element analysis of turbocharger compressor impeller[D]. Shenyang: Northeastern University, 2009.

[2] R .P. Gupta and P. Chandra, Bifurcation analysis of modified Leslie-Gower predator-prey model with Michaelis-Menten type prey harvesting[J]. Math. Anal. Appl., 2013(398): 278-295.

[3] R. P. Gupta. Dynamical Complexity of A Prey-Predator Model with Nonlinear Predator Harvesting[J]. Discete and continuous dynamical systems series B, 2015, 20(2): 423-443.

[4] Y. Zhu, K. Wang. Existence and global attractively of positive periodic solution for a predator-prey model with modified Leslie-Gower Holling type II schemes[J]. Math.And.Appl., 2011(384): 400-408.

[5] K. Salch. A Ratio-dependent Predator-prey System with Quadratic Predator-Harvesting[J]. Journal of Axian Basic and Applied Sciences, 2012(4): 21-25.

[6] D. Xiao, W. Li, M. Han. Dynamics in a ratio-dependent predator-prey model with predator harvesting[J]. Math. Anal. Appl., 2006(324): 14-29.

[7] P. Lenzini, J. Rebaza, Nonconstant predator harvesting on ratio-dependent predator-prey models[J]. Appl. Math. sci., 2010(4): 791-803.

[8] G. J. Peng, Y. L. Jiang, C. P. Li. Bifurcations of a Holling-type II predator-prey system with constant rate harvesting $[\mathrm{J}]$. Int. J. Bifurc. Chaos Appl.Sci.Eng., 2009(19): 5499-2514

[9] R.P. Gupta, P. Chandra. Bifurcation analysis of modified Leslie-Gower predator-prey model with Michaelis-Menten type prey harvesting[J]. Math. Anal. Appl. 2013( 398) 278-295.

\section{Author Profile}

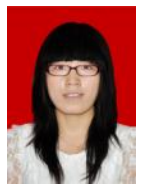

Ji Zhang is reading the M.S. degrees in College of Science from Tianjin University of Technology and Education. 